\title{
COVID-19 vaccines: neutralizing antibodies and the alum advantage
}

\author{
Peter J. Hotez ${ }^{1,2,3,4 凶}$, David B. Corry ${ }^{5,6 凶}$, Ulrich Strych $\mathbb{D}^{1,2 \bowtie}$ and \\ Maria Elena Bottazzi(ii) 1,2,3凶
}

\author{
Achieving high levels of neutralizing antibodies to the spike protein of SARS-CoV- 2 in a safe \\ manner is likely to be crucial for an effective vaccine. Here, we propose that aluminium-based \\ adjuvants might hold the key to this.
}

'Texas Children's Center for Vaccine Development, Houston, TX, USA.

${ }^{2}$ Departments of Pediatrics and Molecular Virology and Microbiology, National School of Tropical Medicine, Baylor College of Medicine, Houston, TX, USA.

${ }^{3}$ Department of Biology, Baylor University, Waco, TX, USA.

${ }^{4}$ Hagler Institute for Advanced Study at Texas A\&M University, College Station, TX, USA.

${ }^{5}$ Departments of Medicine (Immunology, Allergy and Rheumatology) and Pathology \& Immunology, Baylor College of Medicine, Houston, TX, USA.

${ }^{6}$ Michael E. DeBakey VA Center for Translational Research in Inflammatory Diseases, Houston, TX, USA.

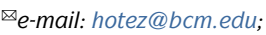
dcorry@bcm.edu; strych@bcm.edu; bottazzi@bcm.edu https://doi.org/10.1038/ s41577-020-0358-6
Scientists are racing to develop safe and effective vaccines to prevent COVID-19, with at least a dozen candidate vaccines already in the clinic or soon to enter clinical development. New studies from animal trials and a human trial already indicate some potential trends, with an emphasis on achieving high levels of neutralizing antibodies. For example, vaccine-elicited pseudovirus-neutralizing antibody titres correlated with protective efficacy in rhesus macaques ${ }^{1}$. Neutralizing antibodies to the coronavirus spike $(S)$ protein might eventually emerge as an immune correlate.

So far, two of the most promising results in terms of inducing high titres of S protein-neutralizing antibodies in preclinical models have been achieved with traditional vaccine approaches. A chemically inactivated virus vaccine (PiCoVacc) and a recombinant proteinbased vaccine (CoV-RBD219N1) were recently shown to elicit high levels of protective immunity in rhesus macaques or in mice against homologous virus challenge with SARS-CoV-2 or SARS-CoV, respectively ${ }^{2,3}$. All macaques that received $\mathrm{PiCoVacc}$ had no detectable virus in their pharynx or lungs after SARS-CoV-2 challenge $^{2}$, and mice that received CoV-RBD219N1 showed $100 \%$ survival after SARS-CoV challenge, compared with $0 \%$ survival in the adjuvant-only controls ${ }^{3}$.

PiCoVacc induced neutralizing antibody titres in the thousands in mice and of up to 400 in rhesus macaques ${ }^{2}$. The macaques also exhibited end point elution titres against the receptor-binding domain (RBD) of the SARS-CoV-2S protein that exceeded 10,000 (REF. ${ }^{2}$ ). Similarly, mice vaccinated with CoV-RBD219N1, based on the recombinant RBD protein of SARS-CoV, which is now investigated as a COVID-19 vaccine candidate, exhibited virus-neutralizing antibody titres between 640 and 1,280 upon SARS-CoV homologous viral challenge ${ }^{3}$.

By contrast, two adenovirus-vectored vaccines were recently shown to induce more modest levels of neutralizing antibodies ${ }^{4,5}$. The chimpanzee adenovirus-vectored COVID-19 vaccine, ChAdOx1 nCoV-19, elicited neutralizing antibody titres in the 5-40 range in rhesus macaques ${ }^{4}$ and was associated with partial protection; three of the six vaccinated macaques developed increased respiratory rates after viral challenge and there was no difference in the quantity of nasal viral RNA between the vaccinated and control groups ${ }^{4}$. In the first reported phase I trial, a human adenovirus 5-vectored COVID-19 vaccine induced both pseudovirus-neutralizing and live-virus-neutralizing antibody titres in a similar range in healthy adults 28 days post-vaccination ${ }^{5}$. Both adenovirus-vectored vaccines induced neutralizing antibody titres that fell significantly below the range reported in human convalescent plasma, whereas initial reports suggested that a group of DNA-based COVID-19 vaccines induced neutralizing antibody titres in macaques that were comparable to those in human convalescent plasma ${ }^{1,6}$.

Therefore, an emerging story in COVID-19 vaccine development is the potential importance of inducing high levels of neutralizing antibodies to the $S$ protein or its RBD. As clinical testing evolves, it will be essential to confirm the role of such antibodies as immune correlates of protection and whether such findings could be used to prioritize different vaccine candidates for clinical trials. This will require harmonizing neutralizing antibody testing results (against both live SARS-CoV-2 and pseudoviruses) across both clinical trials and non-human primate studies in order to allow accurate comparisons.

A key finding so far is that aluminium adjuvant formulations, such as those used for PiCoVacc and CoV-RBD219N1, appear to promote high titres of neutralizing antibody. The actual mechanisms by which aluminium induces high levels of neutralizing antibodies remain elusive ${ }^{7}$. Early studies indicated that aluminium formulations form intramuscular or subcutaneous depots associated with slow antigen release, but more recent studies propose that they may promote activation and trafficking of antigen-presenting cells to lymphoid tissues ${ }^{7}$. Aluminium formulations also trigger the inflammasome and complement activation ${ }^{7}$. A more recent hypothesis derives from links noted between aluminium and blood clotting based on aluminium's 
Box 1 | US-licensed vaccines containing aluminium

- Anthrax

- Hepatitis A

- Hepatitis B

- Human papillomavirus (HPV)

- Diphtheria-pertussis-tetanus (DPT and TdaP)

- Haemophilus influenzae type b

- Japanese encephalitis

- Pneumococcal conjugate vaccines

original medicinal use as a styptic agent, usually formulated as aluminium potassium sulfate (alum). By promoting blood clotting, alum foments cleavage of the clotting protein fibrinogen, which was shown to induce allergic inflammation in disorders such as asthma ${ }^{8}$. Soon after its discovery, alum was shown to specifically induce the development of $\mathrm{T}$ helper $2\left(\mathrm{~T}_{\mathrm{H}} 2\right)$ cells that strongly drive allergic inflammation. Importantly, $\mathrm{T}_{\mathrm{H}} 2$ cells are particularly effective at promoting humoral immunity while at the same time reducing the potential for bystander tissue injury, which is a hallmark of strong $\mathrm{T}_{\mathrm{H}}$ 17-type responses.

A potential concern about the use of aluminium adjuvants is based on the claim that $\mathrm{T}_{\mathrm{H}}$ 2-type immune responses might promote vaccine-enhanced respiratory disease (VAERD) ${ }^{9}$. However, no evidence for this was seen in the studies with the two aluminium-adjuvanted coronavirus vaccines described above ${ }^{2,3}$. Instead, aluminium formulations may actually reduce immunopathology compared with unadjuvanted coronavirus vaccines ${ }^{10}$. Such observations have stimulated in-depth review and careful reading of the scientific literature, with the intent to ensure interpretations are not made based on dogma or on opinions mistakenly concluding that eosinophils arise only through $\mathrm{T}_{\mathrm{H}}$ 2-type responses. They highlight robust evidence that VAERD was first observed in experimental animals with virus-vectored vaccines that induced elevated levels of IL- 6 and point to the potential role of $\mathrm{T}_{\mathrm{H}} 17$ cell responses that promote recruitment of eosinophils from the bone marrow and extravasation into host tissues ${ }^{10}$. Similarly, $\mathrm{T}_{\mathrm{H}} 17$ cell responses are likely responsible for the enhanced immunopathology of severe asthma, inflammatory bowel disease and other conditions ${ }^{10}$. Still, another assertion that aluminium-adjuvanted vaccines induce autism or other chronic illnesses has been thoroughly discredited.

In addition to the immunological advantages it has over other adjuvants, aluminium has a proven, unmatched track record for safety and efficacy dating back to the 1930s and 1940s (BOX 1). Traditional vaccine platforms and the use of aluminium as adjuvant have so far been mostly omitted from consideration as part of the US 'Operation Warp Speed' COVID-19 vaccine programme, even though they may represent our most promising vaccine candidates and formulations in terms of eliciting protective immunity without inducing immunopathology. Aluminium offers promise as a key adjuvant for COVID-19 vaccines comprised of traditional inactivated viruses and recombinant proteins. Aluminium hydroxide (Alhydrogel) is the adjuvant for CoV-RBD219N1, while the type of aluminium used in PiCoVacc has not been specified. Moreover, one or more of the vaccines developed by GlaxoSmithKline use aluminium adjuvants, and these may also be made available through high-level agreements for other COVID-19 vaccines.

Over the course of a century, aluminium has become the most widely tested adjuvant component and has proven to be one of the safest, administered to millions of children and adults. Aluminium offers a glide path to inducing high levels of neutralizing antibody, which is increasingly recognized as a cornerstone of the protection afforded by COVID-19 vaccines.

1. $\mathrm{Yu}$, J. et al. DNA vaccine protection against SARS-CoV-2 in rhesus macaques. Science https://doi.org/10.1126/science.abc6284 (2020).

2. Gao, Q. et al. Development of an inactivated vaccine candidate for SARS-CoV-2. Science https://doi.org/10.1126/science.abc1932 (2020).

3. Chen, W.-H. et al. Yeast-expressed SARS-CoV recombinant receptorbinding domain (RBD219-N1) formulated with alum induces protective immunity and reduces immune enhancement. Preprint at bioRxiv https://doi.org/10.1101/2020.05.15.098079 (2020).

4. van Doremalen, $\mathrm{N}$. et al. ChAdOx $1 \mathrm{nCoV}-19$ vaccination prevents SARS-CoV-2 pneumonia in rhesus macaques. Preprint at bioRxiv https://doi.org/10.1101/2020.05.13.093195 (2020).

5. Zhu, F.-C. et al. Safety, tolerability, and immunogenicity of a recombinant adenovirus type-5 vectored COVID-19 vaccine: a dose-escalation, open-label, non-randomised, first-in-human trial. Lancet https://doi.org/10.1016/S0140-6736(20)31208-3 (2020).

6. Robbiani, D. F. et al. Convergent antibody responses to SARS-CoV-2 infection in convalescent individuals. Preprint at bioRxiv https:// doi.org/10.1101/2020.05.13.092619 (2020).

7. Ghimire, T. R. The mechanisms of action of vaccines containing aluminum adjuvants: an in vitro versus in vivo paradigm. Springerplus 4, 181 (2015)

8. Millien, V. O. et al. Airway fibrinogenolysis and the initiation of allergic inflammation. Ann. Am. Thorac. Soc. 11 (Suppl. 5), 277-283 (2014)

9. Graham, B. S et al. Rapid COVID-19 vaccine development. Science 368, 945-946 (2020).

10. Hotez, P. J., Corry, D. B. \& Bottazzi, M. E. COVID-19 vaccine design: the Janus face of immune enhancement. Nat. Rev. Immunol. 20, 347-348 (2020).

Author contributions

P.J.H. wrote the first draft of the article; all authors contributed to editing and discussion of content.

Competing interests

P.J.H. and M.E.B. are investigators leading the development of coronavirus vaccines against SARS-CoV, MERS-CoV and SARS-CoV-2. D.B.C. is a scientific advisor and holds intellectual property in Atropos Therapeutics, LLC. U.S. declares no competing interests. 